\title{
Is there an app for that? Mobile phones and secondary prevention of cardiovascular disease.
}

\author{
NEUBECK, L., CARTLEDGE, S., DAWKES, S. and GALLAGHER, R.
}


Is there an app for that? Mobile phones and secondary prevention of cardiovascular disease

Lis Neubeck

Professor of Nursing | PhD, BA (Hons), RN, FESC

Head of Theme I Long Term Conditions

School of Health and Social Care

Room 4.B.20| Sighthill Campus | Sighthill Court | Sighthill | Edinburgh | EH11 4BN

Mobile: 07491522 050|E:mail: I.neubeck@napier.ac.uk

Susie Cartledge

PhD (Candidate) | BN (Hons)

Department of Epidemiology and Preventive Medicine

Monash University

Level 3, 553 St Kilda Road | Melbourne | VIC | 3004 | Australia

Mobile: +61438263164 | E:mail susie.cartledge@monash.edu

Susan Dawkes

Associate Professor / Director of Learning and Teaching |RN, PhD, MSc, BSc

School of Health and Social Care

Edinburgh Napier University

Room 4.B.28| Sighthill Campus | Sighthill Court | Sighthill | Edinburgh | EH11 4BN

Mobile: 07718969146|E:mail: s.dawkes@napier.ac.uk

Robyn Gallagher

Professor of Nursing RN PhD FAHA FESC

Charles Perkins Centre I Sydney Nursing School

THE UNIVERSITY OF SYDNEY

Room 2210, Level 2, Building D17 | The University of Sydney | NSW | 2006

Adjunct Professor, Faculty of Health, University of Technology, Sydney

robyn.gallagher@sydney.edu.au 


\section{ABSTRACT}

\section{Purpose of review}

Advances in technology coupled with increased penetration of mobile phones and smart devices is rapidly changing health care delivery. Mobile phone applications ('apps'), text messages, Internet platforms used alone or in combination are now providing interventions targeting people with multiple cardiovascular risk factors. This article will review the emerging evidence of apps and discuss the future of secondary prevention via mobile phones.

\section{Recent findings}

Seven recent randomised controlled trials used text messages or apps for six to 12 months, with or without differing combinations of other technology platforms. All studies demonstrated at least one positive improvement to cardiovascular risk factors measured in cardiac and diabetes populations. When measured, acceptability of the intervention was high.

\section{Summary}

Mobile apps and technology can deliver positive outcomes for managing cardiovascular risk factors. However, due to the complexity of combination interventions, it is difficult to determine the active ingredient. A future challenge for researchers and clinicians will be responding quickly to these rapidly evolving interventions to ensure the delivery of effective outcomes that are evidence-based.

\section{KEYWORDS}

cardiovascular disease; secondary prevention; telehealth; text messaging; mobile applications 


\section{INTRODUCTION}

Cardiac rehabilitation and secondary prevention programmes are recommended in international guidelines for people recovering from a cardiac event [1]. These programmes aim to reduce multiple cardiovascular disease (CVD) risk factors, and ensure uptake of cardioprotective medications. They have been demonstrated to reduce morbidity and mortality from CVD [2]. However, such programmes are typically delivered in an out-patient setting, with group-based exercise, and are time-limited [3]. Globally, reported participation rates are low, with the majority of cardiac event survivors failing to meet key CVD risk reduction targets [4]. Many barriers exist to referral, uptake, and completion, which have been widely documented [5]. Amongst the barriers, distance, timing of programmes, and unwillingness to participate in a group, are frequently cited [5]. With the rapid increase in mobile phone ownership, mobile phone enabled programmes are suggested as one way to address these barriers.

Mobile applications or 'apps' are computer programs which operate on a smartphone, tablet, or other mobile device. They have a wide range of intended uses, from service provision, such as banking apps and travel booking, to social media and games. Mobile apps have become so commonplace in cardiovascular health that both the European Society of Cardiology and the American Heart Association have recently released position papers on their use $[6,7]$. Both organisations comment on the great potential of mobile apps to transform cardiovascular health, but urge caution about the quality of widely available apps. This is not surprising, given the rapid development of apps. From June 2015 to January 2017 the number of available apps in Apple's app store alone rose from 1.5 million to 2.2 million [8]. The largest app store, Google Play, has over 2.5 million apps available [9].

Research involving apps has been considerably slower to evolve, and the majority of trials test a mobile phone based intervention aimed at improving a single risk factor, e.g. weight loss. However, there is emerging evidence that mobile apps can increase uptake and improve multiple CVD risk factors for people with CVD. Overall, seven studies have published results of randomised controlled trials (RCT) of mobile phone apps which provide multiple CVD risk factor reduction (Table 1) [10-15]. These studies were published between 2012-2016 and used a variety of interventions from text messaging to combinations of mobile apps, text messages, and Internet pages.

\section{Studies delivering interventions via mobile phone}

The simplest mobile phone based interventions are text messages. Although several studies have used text messages as part of the intervention, only one study, the Tobacco, Exercise and Diet Messages RCT (TEXT-ME) has investigated text messaging without other components (e.g. supportive website) [11]. This single-blind, parallel group study recruited 710 participants who had either had a myocardial infarction, or had coronary heart disease confirmed on angiogram. The participants were mostly male (82\%), and mean age was $58 \pm 9.2$ years. Both groups participated in usual care, which included community follow up, and the majority were referred to cardiac rehabilitation. In addition, those in the 
intervention group ( $n=352$ ) received text messages four times a week for six months. The messages had been developed and tested to give simple, positive healthy information around key CVD risk reduction behaviours and medication adherence [16]. The messages were automated and tailored to the individuals' baseline characteristics; for example, nonsmokers did not receive smoking cessation messages. The programme was not interactive. At six months, the primary endpoint of low density lipoprotein was $76 \mathrm{mg} / \mathrm{dl}$ in the intervention group, compared to $84 \mathrm{mg} / \mathrm{dl}$ in the usual care group, a mean difference of $8 \mathrm{mg} / \mathrm{dl}$, which was statistically significant $(\mathrm{p}=0.04)$. Importantly, there were improvements in multiple CV risk factors in the intervention group. There were some interesting observations about the study from the qualitative evaluation. Firstly, although all participants were advised that the system was automated and one-directional, 403 text messages were received by the investigators from 116 unique mobile phone numbers [17]. It also appeared that when participants received the text messages they imagined that the research assistant who had recruited them was sending the messages personally. Importantly, the majority of the participants found the intervention acceptable, and many would have liked the intervention to continue past the six months of the study [17].

The other six studies used a combination of an Internet platform and either text messages, or biometric monitoring, or a mobile app. Using multiple elements makes it challenging to unpick which of these elements are the primary driver of behaviour change. The study by Blasco et al [10] was a single-blind RCT of 203 acute coronary syndrome survivors. Both groups received lifestyle counselling and the usual care of their treating physician. Those in the intervention group uploaded their weight, heart rate, blood pressure, lipids and glucose via their mobile phone, which were sent to a web interface. A cardiologist reviewed the data in the web interface, and made tailored recommendations, which were sent by text message. At 12 months, the intervention group, compared to the control group, had better achievement of treatment goals for blood pressure (62.1\% vs $42.9 \%, p=0.012)$, and glycated haemoglobin $A_{1 c}(86.4 \%$ vs $54.2 \%, p=0.018)$. They also had lower body mass index ($0.77 \mathrm{~kg} / \mathrm{m} 2 \mathrm{vs}+0.29 \mathrm{~kg} / \mathrm{m} 2), p=0.005)$. However, there were no differences in low density lipoprotein levels or smoking cessation [10].

Another study that involved a monitoring health professional, delivering an intervention via a mobile phone was conducted in Finland by Karhula at al [18]. This study randomised 267 participants with coronary heart disease, and 250 participants with diabetes to a selfmonitoring intervention, where the participants were asked to upload blood glucose, blood pressure, weight and steps to a mobile phone, which then automatically transferred the data to the patient's personal health record. The health professional then called the participant on the mobile phone every 4-6 weeks to discuss the information that had been uploaded. The primary endpoint of the study was health related quality of life, however no difference in outcome was observed in either group. There were observed differences in the waist circumference between the coronary heart disease groups, and the diabetes groups, which led the authors to conclude that the intervention may work differently and be of more benefit to participants with diabetes [18]. However, although the intervention is described as a mobile phone intervention and appears to include text messaging, the text 
messages went to the health care professional, so the primary potential for the intervention to promote behaviour change was really through 4-6 weekly telephone calls.

The SUPPORT study [12] focused on increasing adherence to an anti-platelet drug, but also aimed to affect multiple cardiovascular risk factors in 174 individuals after a myocardial infarction. Both intervention and control groups received cardiac rehabilitation, as per the Swedish national standard. Both groups were also provided with a drug adherence diary app on their smartphone, which allowed the participants to record their intake of the antiplatelet drug, and both groups were sent a text message reminder if they did not record taking the drug within the prescribed timeframe. In addition, the intervention group received educational messages within the app, tailored to their individual record. Within the apps, the intervention group could access four educational modules, including extended drug adherence, exercise, weight management and smoking cessation. An automated feedback logic was developed to ensure the messages were tailored, but the data that patients recorded was not monitored. When a message was generated in the app, a text message was also sent to alert the participant. At six months, the intervention group achieved greater medication adherence, compared to the control group (non- adherence score 16.6 vs $22.8, p=0.025$ ) [12].

The HEART (Heart Exercise And Remote Technologies) study [15] involved a personalized, automated package of text messages and a secure internet site. This site included video messages aimed at increasing exercise behaviour, delivered over 24 weeks. Participants with coronary heart disease $(n=171)$ were randomised to usual care, or the HEART intervention. The primary outcome was peak oxygen uptake $\left(\mathrm{PVO}_{2}\right)$. At 24 weeks there were no differences in $\mathrm{PVO}_{2}$ between the two groups (difference $-0.21 \mathrm{ml} \mathrm{kg}^{-1} \mathrm{~min}^{-1}, 95 \% \mathrm{Cl}$ : $-1.1,0.7 ; p=0.65)$. However, there were significant improvements in outcomes, including leisure time physical activity (difference $110.2 \mathrm{~min} /$ week, $95 \% \mathrm{Cl}:-0.8,221.3 ; p=0.05$ ) and walking (difference $151.4 \mathrm{~min} /$ week, 95\% Cl: 27.6, 275.2; $p=0.02$ ).

Similarly, The Text4Heart study [13], which was conducted by the same investigators as the HEART study, also included daily text messages and a supporting website over a 24-week period. The messages were fully automated and underpinned by social cognitive theory. The trial included 123 participants with coronary heart disease, randomised to usual care, or the intervention group. The primary outcome was adherence to healthy lifestyle behaviors measured using a self-reported composite health behavior score $(\geq 3)$ at 3 and 6 months. A significant treatment effect in favor of the intervention was observed for the primary outcome at 3 months (AOR 2.55, 95\% Cl 1.12-5.84; $P=.03$ ), but not at 6 months (AOR 1.93, $95 \% \mathrm{Cl} 0.83-4.53 ; P=.13)$. Of note, $85 \%$ of people reported reading all the text messages they were sent, while visits to the supporting website varied from 0 to 100 per individual [13].

The final study is the Care Assessment Platform [14]. This was a randomised controlled trial of traditional cardiac rehabilitation compared to an intervention group using a smartphone cardiac rehabilitation app providing daily SMS messages, multimedia education topics, relaxation audio files, and a light to moderate physical activity program. Participants were able to set and monitor goals and the app included a step counter and exercise diary. Trial 
results demonstrated uptake of cardiac rehabilitation was much higher in the intervention group. Importantly, the intervention group had significantly higher adherence and completion than the traditional cardiac rehabilitation group. Both the intervention group and traditional cardiac rehabilitation group significantly improved their 6-minute walk test distance ( $537 \pm 86-584 \pm 99 \mathrm{~m}$ vs $510 \pm 77-570 \pm 80 \mathrm{~m}$ ), emotional state, and quality of life; and lost non-significant amounts of weight. The study demonstrated a smartphone app can achieve equivalent improvements in secondary prevention outcomes as traditional cardiac rehabilitation, and importantly had significantly higher uptake ( $80 \%$ vs $62 \%$ ), adherence ( $94 \%$ vs $68 \%$ ) and completion ( $80 \%$ vs $47 \%$ ) rates than a traditional cardiac rehabilitation group $(p<0.05)$.

\section{The future of secondary prevention via mobile phones}

The complexity of these interventions makes identifying the active ingredient and appropriate dose challenging. Mixed methods study design, and process evaluations will help to improve understanding of what really matters. Even in the TEXT-ME study, where the intervention appeared to be a simple one-directional text message, the qualitative component revealed that people tend to assign human characteristics to the intervention, believing that the automated messages were sent by the friendly research assistant who had recruited them [17].

As technology evolves this understanding will become ever more important. Wearable trackers that monitor physical activity have been shown to provide valid and reliable information for people with coronary heart disease [19]. To extend this further, the Internet of Things-where connected devices talk to each other to inform health care decisions- may become the secondary prevention tools of the future. An individual might have sensors at home or in the car that detect changes in heart rate or breathing, or subtle changes in skin colour that suggest need for a change in medication, or a reminder to take medication. Common digital assistants such as Apple's Siri could be adapted for health purposes so that an algorithm prompts correct choices in health behaviours. Our biggest challenge as researchers and clinicians will be responding quickly enough to ensure that the evolving technologies are evidence-based and that we understand the benefits, or lack thereof, of the tools that are available.

\section{CONCLUSION}

Evidence for mobile phone delivered secondary prevention is emerging, and of seven RCT's, six show improvements in cardiovascular risk factors, when compared to control. However, the diversity of the interventions makes it challenging to understand which ingredient matters most when it comes to recommending mobile phone interventions. In addition, long term outcomes are currently unknown. As there are a multitude of apps currently available, it is also important that consumers can make informed choices. The evidencebase for the apps needs to be robust, credible, clear and unambiguous. Health-care bodies could regulate CVD apps or organisations such as heart foundations could provide endorsement for them., but volume of available apps makes this challenging. It may be 
more feasible to provide consumers with tools to make informed decisions around choosing evidence-based apps that provide the best outcomes than to try to accredit individual apps.

- There is emerging evidence that mobile phones interventions improve CVD risk factors

- It is hard to determine the 'active ingredient' of mobile apps

- The long term benefit of $\mathrm{m}$-health is unknown

\section{Acknowledgements}

We would like to thank Sheena Moffat from Edinburgh Napier University for her help with searching for papers.

\section{Financial support and sponsorship}

No funding was received for this paper.

\section{Conflicts of interest}

The authors have no conflicts of interest in relation to this paper 


\section{REFERENCES}

1. European Society of Cardiology, CVD Prevention in Clinical Practice (European Guidelines on). available at http://www.escardio.org/Guidelines/Clinical-Practice-Guidelines/CVDPrevention-in-clinical-practice-European-Guidelines-on accessed 7th November 1016, 2016.

2. Anderson, L., et al., Exercise-based cardiac rehabilitation for coronary heart disease: Cochrane systematic review and meta-analysis. Journal of the American College of Cardiology, 2016. 67(1): p. 1-12.

3. Briffa, T., et al., An integrated and coordinated approach to preventing recurrent coronary heart disease events in Australia. Policy statement from the Australian Cardiovascular Health and Rehabilitation Association. Med J Aust, 2009. 190(12): p. 683 - 686.

4. Redfern, J., et al., Prescription of secondary prevention medications, lifestyle advice, and referral to rehabilitation among acute coronary syndrome inpatients: results from a large prospective audit in Australia and New Zealand. Heart, 2014: p. heartjnl-2013-305296.

5. Neubeck, L., et al., Participating in cardiac rehabilitation: A systematic review and metasynthesis of qualitative data European Journal of Cardiovascular Prevention \& Rehabilitation, 2011. 19(3): p. 194-503.

6. Cowie, M.R., et al., e-Health: a position statement of the European Society of Cardiology. European heart journal, 2016. 37(1): p. 63-66.

7. Burke, L., et al., on behalf of the American Heart Association Publications Committee of the Council on Epidemiology and Prevention, Behavior Change Committee of the Council on Cardiometabolic Health, Council on Cardiovascular and Stroke Nursing, Council on Functional Genomics and Translational Biology, Council on Quality of Care and Outcomes Research, and Stroke Council. Current science on consumer use of mobile health for cardiovascular disease prevention: a scientific statement from the American Heart Association [published online ahead of print August 13, 2015]. Circulation. doi, 2015. 10.

8. Statista, Number of available apps in the apple app store. https://www.statista.com/statistics/263795/number-of-available-apps-in-the-apple-appstore/ accessed 10/02/2017, 2017.

9. Statista, Number of available applications available in the google play store. https://www.statista.com/statistics/266210/number-of-available-applications-in-thegoogle-play-store/ accessed 10/02/2017, 2016.

10. Blasco, A., et al., Evaluation of a telemedicine service for the secondary prevention of coronary artery disease. Journal of cardiopulmonary rehabilitation and prevention, 2012. 32(1): p. 25-31.

11. Chow, C.K., et al., Effect of lifestyle-focused text messaging on risk factor modification in patients with coronary heart disease: a randomized clinical trial. Jama, 2015. 314(12): p. 1255-1263.**

12. Johnston, N., et al., Effects of interactive patient smartphone support app on drug adherence and lifestyle changes in myocardial infarction patients: A randomized study. American heart journal, 2016. 178: p. 85-94.

13. Dale, L.P., et al., Text message and internet support for coronary heart disease selfmanagement: results from the Text4Heart randomized controlled trial. Journal of medical Internet research, 2015. 17(10).

14. Varnfield, M., et al., Smartphone-based home care model improved use of cardiac rehabilitation in postmyocardial infarction patients: results from a randomised controlled trial. Heart, 2014: p. heartjnl-2014-305783.*

15. Maddison, R., et al., A mobile phone intervention increases physical activity in people with cardiovascular disease: Results from the HEART randomized controlled trial. European journal of preventive cardiology, 2015. 22(6): p. 701-709. 
16. Redfern, J., et al., Development of a set of mobile phone text messages designed for prevention of recurrent cardiovascular events. European journal of preventive cardiology, 2014. 21(4): p. 492-499.

17. Redfern, J., et al., Factors Influencing Engagement, Perceived Usefulness and Behavioral Mechanisms Associated with a Text Message Support Program. PloS one, 2016. 11(10): p. e0163929.*

18. Karhula, T., et al., Telemonitoring and mobile phone-based health coaching among finnish diabetic and heart disease patients: randomized controlled trial. Journal of medical Internet research, 2015. 17(6): p. e153.

19. Alharbi, M., et al., Validation of Fitbit-Flex as a measure of free-living physical activity in a community-based phase III cardiac rehabilitation population. European journal of preventive cardiology, 2016: p. 2047487316634883.

${ }^{* *}$ Chow et al TEXT ME was a large RCT with a simple intervention which had significant impact of CVD risk factors. This paper is particularly notable for the simplicity of the intervention, it's potential scalability and generalisability.

* Redfern et al produced interesting insights into the mechanisms and context of the TEXT ME RCT and begins to explain some of the reasons this trial may have been successful.

*Varnfield et al undertook the world first mobile app in CVD risk factor reduction. It is most notable for the increased participation and completion rates in the group that undertook the mobile intervention. 